\title{
Composição, abundância e notas sobre a ecologia de espécies de larvas de lepidópteros associadas a cinco espécies de plantas hospedeiras no Parque Nacional da Restinga de Jurubatiba, RJ
}

\author{
Ricardo F. Monteiro ${ }^{1}$, Margarete V. Macedo ${ }^{1}$, Milena de S. Nascimento ${ }^{1} \&$ Rafael S. F. Cury $^{1}$
}

${ }^{1}$ Laboratório de Ecologia de Insetos, Depto. de Ecologia, IB, UFRJ. Caixa Postal 68020, 21941-590, Rio de Janeiro-RJ, Brasil. monteiro@biologia.ufrj.br, mvmacedo@biologia.ufrj.br, nascimentoms@biologia.ufrj.br, rafaelcury@terra.com.br

\begin{abstract}
Composition, abundance and notes on ecology of lepidopteran larvae associated with five plant species in Parque Nacional da Restinga de Jurubatiba, RJ. In order to describe the species composition, temporal occurrence and ecological aspects of larvae associated with five plant species: Erythroxylum ovalifolium and E. subsessile (Erythroxylaceae), Manilkara subsericea (Sapotaceae), Protium icicariba and P. heptaphyllum (Burseraceae), 1.680 plants were bimonthly surveyed from July of 2003 to August of 2005 in the Parque Nacional da Restinga de Jurubatiba (PNRJ). Thirty two species of Lepidoptera were reared from these five host plants, and the most abundant four species belonged to the Elachistidae. Manilkara subsericea showed the greatest number of Lepidoptera species associated, 14 species, followed by E. ovalifolium (10 species), P. icicariba (seven species), E. subsessile (six species) and P. heptaphyllum (two species). Only one out of the 32 species of Lepidoptera was gregarious and 56\% used any kind of shelter to get food or for defence. Six Lepidoptera species (27\%) were polyphagous, three (14\%) oligophagous and $13(59 \%)$ had just only one host plant species recorded. The most abundant species of moths exhibitted two reproductives periods in the year, predominantly in winter and autumn. Abiotic factors, such as strong insolation during spring and summer in the open scrub vegetation of restinga, may be very important in producing this temporal pattern of insect reproduction, but we cannot neglect biotic factors like natural enemies.
\end{abstract}

KEYWORDS. Elachistidae; host record; insect-plant interactions; specificity.

RESUMO. Composição, abundância e notas sobre a ecologia de espécies de larvas de lepidópteros associadas a cinco espécies de plantas no Parque Nacional da Restinga de Jurubatiba, RJ. Com o objetivo de descrever a composição, ocorrência temporal e aspectos ecológicos das espécies de larvas de Lepidoptera associadas a cinco espécies de plantas: Erythroxylum ovalifolium e E. subsessile (Erythroxylaceae), Manilkara subsericea (Sapotaceae), Protium icicariba e $P$. heptaphyllum (Burseraceae), 1.455 plantas foram vistoriadas durante o período de Julho de 2003 a Agosto de 2005 no Parque Nacional da Restinga de Jurubatiba (PNRJ). Foram encontradas 32 espécies de Lepidoptera associadas a essas cinco espécies de plantas hospedeiras, sendo que as quatro espécies mais abundantes pertenciam à família Elachistidae. Manilkara subsericea apresentou o maior número de espécies de Lepidoptera associadas, 14 espécies, seguida por $E$. ovalifolium, com 10 espécies, P. icicariba, com sete, E. subsessile com seis e P. heptaphyllum com duas espécies de Lepidoptera associadas. Apenas uma das 32 espécies de Lepidoptera criadas apresentava hábito gregário e $56 \%$ utilizavam algum tipo de abrigo para alimentação e/ou defesa. Seis espécies de Lepidoptera identificadas (27\%) eram polífagas, três (14\%) oligófagas e 13 (59\%) tiveram apenas uma única espécie de planta hospedeira registrada. As espécies mais abundantes de mariposas apresentaram dois períodos reprodutivos no ano, predominantemente, no inverno e outono. Fatores abióticos, como a forte insolação na primavera e verão na formação aberta de restinga, podem ser importantes determinando esse padrão temporal de reprodução dos insetos, mas não podemos descartar também fatores bióticos como a pressão de inimigos naturais.

PALAVRAS-CHAVE. Elachistidae; especificidade; interação inseto-planta; registro de hospedeiro.

Para imaturos de insetos fitófagos, a planta hospedeira representa um abrigo contra condições abióticas adversas, inimigos naturais e, ao mesmo tempo, seu recurso alimentar (Southwood 1973). A maioria dos insetos que se alimentam de plantas são oligófagos, utilizando espécies de um mesmo gênero ou família, mas não raramente existem espécies que são monófagas, isto é, utilizam somente uma espécie de planta hospedeira (Ehrlich \& Raven 1964), o que torna muito importante o conhecimento de suas plantas hospedeiras.

No Brasil, os registros de plantas hospedeiras de insetos fitófagos e informações de sua biologia e comportamento têm como fonte principal estudos pontuais ligados a alguns táxons de insetos, em geral, relacionados a pragas agrícolas ou vetores de doenças, que foram resumidos nos volumes clássicos de Costa Lima (1945, 1950) e de D'Araújo e Silva et al. (1968). Muitos registros são veiculados, apenas, em periódicos não indexados, relatórios de instituições de pesquisa ou ainda em dissertações.

Recentemente, vários estudos têm apresentado numerosos dados sobre insetos associados a plantas no cerrado do Brasil Central. Neles há registros de plantas hospedeiras de aproximadamente 150 espécies de Lepidoptera (Diniz \& Morais 1995; Diniz \& Morais 1997; Andrade et al. 1999; Diniz et al. 2000;). Além da composição de insetos nas plantas hospedeiras, estes estudos têm levantado também aspectos sobre a riqueza, especificidade e raridade das espécies de 
insetos fitófagos no cerrado (Price et al. 1995; Pinheiro et al. 2002). Entretanto, dados disponíveis sobre insetos associados a grupos representativos de plantas em outros ecossistemas brasileiros são ainda relativamente escassos.

Nessa última década surgiram novas contribuições sobre a biologia e a ecologia de lepidópteros de restingas da região sudeste (por exemplo, Monteiro \& Macedo 2000, Monteiro \& Becker 2002, Flinte et al. 2006). Monteiro et al. (2004) apresentam um levantamento pioneiro, mas preliminar ainda, sobre as espécies de Lepidoptera da Restinga de Jurubatiba, porém quase nada é conhecido sobre as plantas hospedeiras desses insetos. Nesse trabalho procurou-se averiguar a composição de espécies de larvas de Lepidoptera associadas a cinco espécies de plantas hospedeiras abundantes no Parque Nacional da Restinga de Jurubatiba (PNRJ) e descrever aspectos gerais da morfologia, comportamento e especificidade dessas larvas.

\section{MATERIALE MÉTODOS}

O trabalho foi realizado no Parque Nacional da Restinga de Jurubatiba (PNRJ), localizado no litoral norte do estado do Rio de Janeiro (Brasil), em uma planície arenosa com vegetação distribuída em moitas, mas com presença também de mata periodicamente inundável (Araújo et al. 1998).

Nessa região, o verão é quente e chuvoso, com temperatura média de $27,9^{\circ} \mathrm{C}$ e precipitação mensal média de $97,7 \mathrm{~mm}$, enquanto que nos meses de inverno, a temperatura média é de $23,7^{\circ} \mathrm{C}$ e a precipitação média cai para $49,2 \mathrm{~mm}$ (Flinte \& Macêdo 2004).

As espécies de plantas escolhidas para averiguação dos insetos fitófagos são arbustos que apresentam grande abundância e ampla distribuição no PNRJ (Araújo et al. 2004): Erythroxylum ovalifolium Peyr. e Erythroxylum subsessile (Mart.) O.E.Schulz (Erythroxylaceae), Manilkara subsericea (Mart.) Dubard (Sapotaceae), Protium icicariba (DC.) March. e Protium heptaphyllum (Aubl.) March (Burseraceae). Outras espécies desses três gêneros ocorrem no PNRJ, porém em densidades bastante baixas (Araújo et al. 2004).

Quinze indivíduos de E. ovalifolium, E. subsessile e $M$. subsericea foram vistoriados, mensalmente, no período de Julho de 2003 a Junho de 2005, na formação arbustiva aberta de Clusia. Em P. icicariba, foram vistoriados, mensalmente, 15 indivíduos na formação de moitas, durante o período de junho de 2004 a agosto de 2005. Protium heptaphyllum teve 15 indivíduos vistoriados mensalmente, de novembro de 2004 a agosto de 2005, também na formação de moita. As plantas, aleatoriamente escolhidas, eram vistoriadas integralmente anotando-se o número de larvas de cada espécie de inseto fitófago. Algumas larvas de cada espécie eram coletadas para criação, observação do seu comportamento e posterior identificação dos adultos. Para a descrição de aspectos de sua morfologia, foram utilizadas larvas de último instar. Observações avulsas também foram realizadas em outras espécies de plantas presentes na região, com o objetivo de registrar outros eventuais hospedeiros.
Em campo, a fenologia das plantas era avaliada observando-se a porcentagem de plantas de cada espécie vistoriada que apresentava, predominantemente, folhas novas, o que indicava a produção ou renovação do recurso alimentar das larvas de lepidópteros estudadas. Os insetos adultos obtidos estão armazenados na coleção de insetos do Departamento de Ecologia, do Instituto de Biologia, da Universidade Federal do Rio de Janeiro.

\section{RESULTADOSEDISCUSSÃO}

A partir dos 1.455 indivíduos vistoriados das cinco espécies de plantas hospedeiras foram encontradas 32 espécies de larvas exofíticas de Lepidoptera. Vinte e duas espécies foram identificadas (Tabela I) e as 10 espécies não identificadas corresponderam àquelas com, apenas, um ou dois indivíduos encontrados que, em muitos casos, morreram por causas conhecidas (parasitóide, por exemplo) ou não.

Manilkara subsericea apresentou o maior número de espécies de Lepidoptera associadas (14), seguida por $E$. ovalifolium (10), e P. icicariba (7). E. subsessile e $P$. heptaphyllum apresentaram, respectivamente, seis e duas espécies associadas (Tabela I).

Diversos fatores relacionados à planta hospedeira podem influenciar a riqueza de insetos fitófagos, como abundância e distribuição geográfica, características morfológicas e estruturais, presença de compostos secundários, disponibilidade e distribuição dos recursos oferecidos pelas plantas hospedeiras, entre outros (Lawton 1982; Leather 1986; Southwood 1986). Muito embora M. subsericea apresente valores relativamente mais baixos de densidade, freqüência e cobertura vegetal, do que as outras quatro espécies estudadas (Araújo et al. 2004), além de ser bastante latescente, apresentou mais espécies de insetos fitófagos associados. Desta forma, sugere-se que outros fatores além desses podem estar relacionados à maior riqueza de espécies de insetos nessa espécie de planta hospedeira. Embora P. heptaphyllum seja, dentre as plantas estudadas, possivelmente aquela com distribuição geográfica mais ampla (Martius et al. 1874), não é tão abundante no PNRJ e foi amostrada por um período de tempo inferior às demais plantas. Esses dois fatores poderiam explicar o baixo número de espécies de Lepidoptera obtido nessa planta.

As ilustrações das larvas de Lepidoptera encontradas (Figs. 1-16), tem o intuito de evidenciar suas características externas gerais, que são complementadas, em seguida, com descrições de seus hábitos, comportamentos, registros de plantas hospedeiras disponíveis na literatura e período de ocorrência. Para quatro espécies, que mostraram grande abundância, são apresentados dados mais detalhados de suas dinâmicas temporais e da variação fenológica de suas plantas hospedeiras (Figs. 17 - 20).

Arctiidae. Trichromia onytes (Cramer, 1777): Coloração verde clara, com presença de muitas cerdas. Alimenta-se na superfície adaxial das folhas. Utiliza as suas cerdas para 

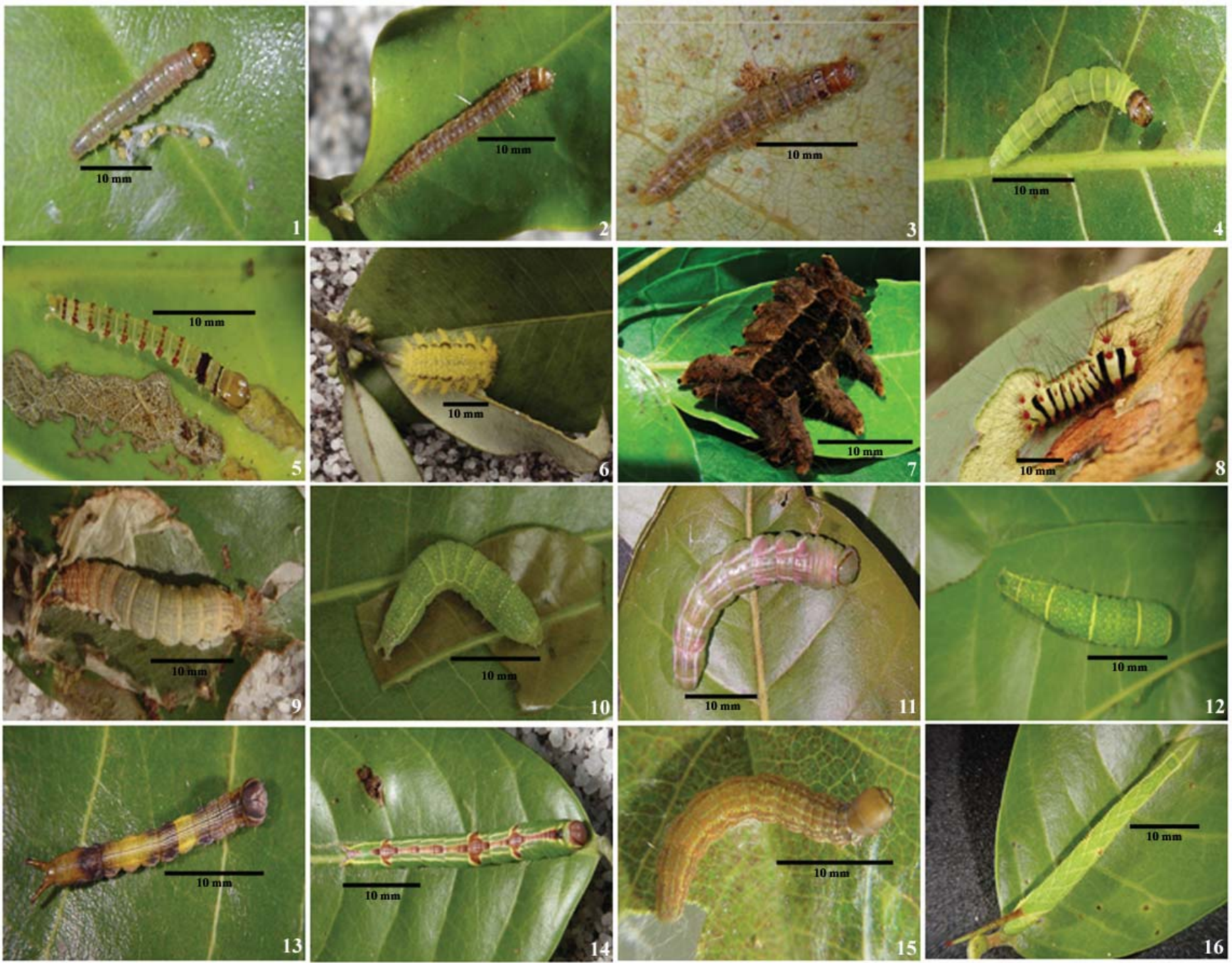

Figs. 1 a 16. Larvas de Lepidoptera das espécies mais representativas obtidas das cinco espécies de plantas hospedeiras vistoriadas no PNRJ. 1: Chlamydastis fragmentella; 2: Stenoma sp.1; 3: Stenoma sp.2; 4: Stenoma sp. 4; 5: Dichomeris sp.1; 6 e 7: polimorfismo de Phobetron hipparchia; 8: Megalopyge lanata; 9: Lacosoma philastria; 10: Paectes albescens; 11: Paectes songoensis; 12: Paectes semicircularis; 13: Rifargia causia; 14: Rifargia distinguenda; 15: Inga inflammata; 16: Protambulyx eurycles.

construir um casulo dentro do qual empupa. Apenas um indivíduo dessa espécie foi registrado, no mês de Abril de 2005. Não foram encontrados registros de suas plantas hospedeiras na literatura.

Elachistidae. Chlamydastis fragmentella (Dognin, 1913) (Fig. 1): Coloração marrom ou verde, de acordo com o instar, com anéis marrons ao longo do corpo. Forma um abrigo unindo duas folhas com a seda por ela produzida. Alimenta-se raspando a superfície da folha, dentro desse abrigo, onde também empupa. Quando molestada, a larva apresenta um comportamento de fuga, jogando-se da folha por um fio-guia. Essa espécie ocorreu durante todo o estudo, mas seus picos de abundância ocorreram nos meses de Setembro de 2003 e 2004 e Abril de 2005 (Fig. 17). Não foram encontrados registros de plantas hospedeiras para essa espécie, porém, segundo Morais et al. (2005), todas as sete espécies de Chlamydastis encontradas em um cerrado de Brasília eram monófagas, sendo quatro delas associadas a diferentes espécies de Sapotaceae. Assim, é provável que haja uma distribuição predominante das espécies de Chlamydastis nesta família latescente de planta.

Stenoma spp. (Figs. 2, 3 e 4): Foram encontradas quatro espécies distintas desse gênero, uma em cada espécie de planta hospedeira (E. ovalifolium, E. subsessile, M. subsericea e $P$. icicariba) com comportamento e morfologia larvais semelhantes. As larvas são de cor marrom, com presença de cerdas ao longo do corpo. Alimentam-se em abrigos, feitos com duas folhas unidas com seda, onde se desenvolvem e empupam. Como estratégia de defesa, apresentam comportamento de fuga, jogando-se da folha, quando perturbada, através de um fio-guia que permite o seu retorno à mesma. Essas espécies foram abundantes, principalmente nos 

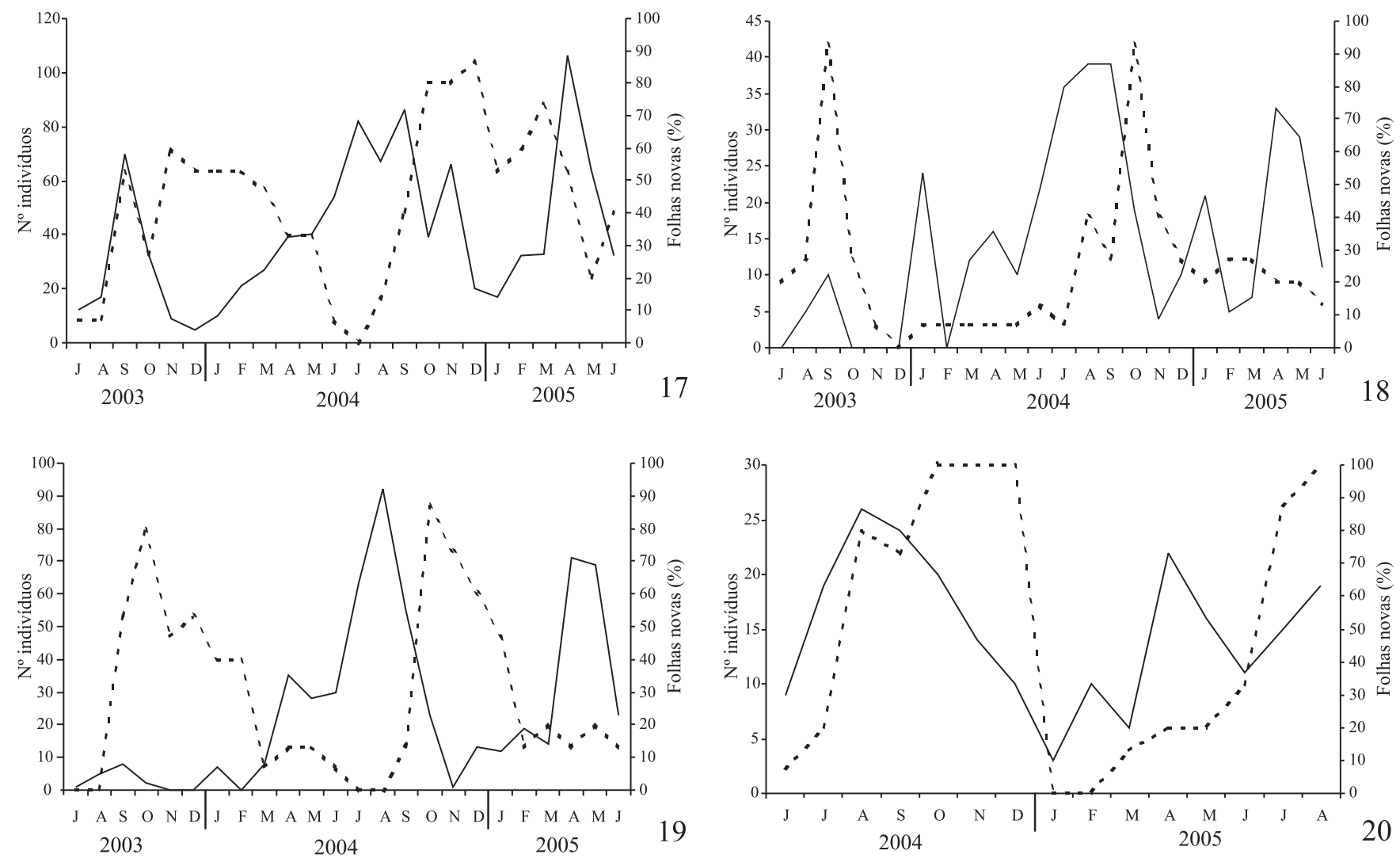

Figs. 17 a 20. Variação mensal do número de larvas das espécies de Lepidoptera mais abundantes ao longo do estudo (linha contínua) e porcentagem de plantas hospedeiras com predominância de folhas novas (linha pontilhada). 17: Chlamydastis fragmentella em Manilkara subsericea; 18: Stenoma sp.1 em Erythroxylum subsessile; 19: Stenoma sp.2 em Erythroxylum ovalifolium; 20: Stenoma sp. 4 em Protium icicariba.

meses de Agosto e Setembro de 2004 e Abril de 2005 (Figs. 18, $19 \mathrm{e} 20)$.

Gelechiidae. Dichomeris spp. (Fig. 5): Foram encontradas duas espécies pertencentes a esse gênero, com características bem semelhantes, porém vivendo em plantas hospedeiras diferentes (E. ovalifolium e E. subsessile). As larvas possuem coloração esverdeada, e anéis de coloração mais escura ao longo de todo o corpo. $\mathrm{O}$ abrigo é feito com uma única folha bem nova (broto), dobrando e unindo as suas pontas com seda, dentro do qual se alimentam e empupam. Quando se sentem ameaçadas, as larvas se jogam da folha através de um fio-guia. Essas espécies ocorreram principalmente nos meses de Setembro e Dezembro de 2003.

Geometridae. Cyclomia ocellata Prout, 1910: Coloração verde-escuro, semelhante ao substrato, sugerindo uma forma de defesa. Apresenta vida livre, e se alimenta na superfície das folhas, onde forma o seu casulo pupal. Essa espécie foi bastante rara, aparecendo apenas dois indivíduos, um no mês de Setembro e outro em Novembro de 2003. Não foram encontrados registros de plantas hospedeiras para essa espécie.
Limacodidae. Phobetron hipparchia (Cramer, 1777) (Figs. 6 e 7): Com o corpo coberto de cerdas, essa espécie apresenta dimorfismo sexual quando adulto, e sua larva pode apresentar coloração amarela, cinza ou marrom, independentemente do sexo. Alimenta-se raspando a superfície das folhas, terminando por construir um casulo de seda no momento final do seu desenvolvimento, onde empupa. Generalista, essa espécie foi registrada no mês de Junho de 2005, alimentando-se em: Erythroxylum ovalifolium, Manilkara subsericea e Allagoptera arenaria (Arecaceae). Existem também registros dessa espécie, em outros biomas, alimentando-se em: Kielmeyera coriacea (Clusiaceae) (Diniz et al. 1999), Anacardium excelsum, Spondias mombin (Anacardiaceae), Chlorophora tinctoria (Moraceae) (Bael et al. 2004), Erythroxylum deciduum e E. tortuosum (Erythroxylaceae) (Diniz \& Morais 1997), Chamaechrista claussemii (Leguminosae) (Diniz \& Morais 1995) e Gliricidia sepium (Leguminosae) (Young 1986). É considerada praga de algumas plantações, como cajueiro, carvalho, goiabeira, palmeira, roseira entre outras (D’Araújo e Silva et al. 1968).

Megalopygidae. Megalopyge lanata (Cramer, 1780) (Fig. 8): Coloração amarela clara com anéis vermelhos ao longo do corpo e muitas cerdas, com as quais a larva constrói seu casulo 
Tabela I. Espécies de Lepidoptera obtidas a partir da criação das Lepidoptera coletadas nas cinco espécies de plantas vistoriadas e sua abundância, no PNRJ, no período entre julho de 2003 e agosto de 2005. A abundância corresponde ao número de indivíduos coletados a cada 100 plantas vistoriadas, uma vez que a quantidade de indivíduos vistoriados foi diferente para as espécies de plantas hospedeiras. Quatro espécies bem mais abundantes que as demais, estão destacadas em negrito.

\begin{tabular}{|c|c|c|c|}
\hline Família & Espécies & Planta Hospedeira & Abundância \\
\hline Arctiidae & Trichromia onytes & M. subsericea & 0,3 \\
\hline Elachistidae & Chlamydastis fragmentella & M. subsericea & 272,5 \\
\hline Elachistidae & Stenoma sp. 1 & E. subsessile & 97,8 \\
\hline Elachistidae & Stenoma sp. 2 & E. ovalifolium & 160,8 \\
\hline Elachistidae & Stenoma sp.3 & M. subsericea & 9,2 \\
\hline Elachistidae & Stenoma sp.4 & P. icicariba & 99,6 \\
\hline Gelechiidae & Dichomeris sp.1 & E. subsessile & 10,0 \\
\hline Gelechiidae & Dichomeris sp. 2 & E. ovalifolium & 7,2 \\
\hline Geometridae & Cyclomia ocellata & E. ovalifolium & 0,6 \\
\hline Limacodidae & Phobetron hipparchia & E. ovalifolium, $M$. subsericea & 0,6 \\
\hline Megalopygidae & Megalopyge lanata & E. ovalifolium, E. subsessile, M. subsericea & 3,1 \\
\hline Mimallonidae & Lacosoma philastria & M. subsericea & 2,5 \\
\hline Noctuidae & Paectes albescens & P. icicariba & 6,2 \\
\hline Noctuidae & Paectes songoensis & P. icicariba, P. heptaphyllum & 2,1 \\
\hline Noctuidae & Paectes semicircularis & P. heptaphyllum & 6,0 \\
\hline Notodontidae & Rifargia causia & P. icicariba & 3,6 \\
\hline Notodontidae & Rifargia distinguenda & P. icicariba & 6,7 \\
\hline Oecophoridae & Inga inflammata & E. ovalifolium, E. subsessile, M. subsericea, $P$. icicariba & 3,6 \\
\hline Pyralidae & Zamagiria laidion & M. subsericea & 6,1 \\
\hline Sphingidae & Erinnyis ello ello & M. subsericea & 0,3 \\
\hline Sphingidae & Protambulyx eurycles & P. icicariba & 4,4 \\
\hline Thyrididae & Banisia myrsusalis & M. subsericea & 1,1 \\
\hline
\end{tabular}

Espécies não identificadas $=10$

pupal. Generalista, foi encontrada em Erythroxylum ovalifolium, E. subsessile e Manilkara subsericea, nos meses de Agosto e Setembro de 2004. Existem ainda registros dessa espécie alimentando-se de Byrsonima sericea (Flinte et al. 2006), B. crassifolia (Malpighiaceae), Kielmeyera coriacea (Clusiaceae) (Diniz \& Morais 1995; Diniz et al. 1999), Swietenia macrophylla (Meliaceae), Roupala montana (Proteaceae) e Rhizophora mangle (Rhizophoraceae) (Janzen \& Hallwachs 2006). D'Araújo e Silva et al. (1968) registraram essa espécie alimentando-se de folhas de abacateiro, cafeeiro, cajueiro, carvalho, cedrinho, laranjeira, limoeiro, entre outras espécies economicamente importantes.

Mimallonidae. Lacosoma philastria Schaus, 1928 (Fig. 9): Possui coloração marrom e constrói um abrigo resistente, enrolando uma folha ao redor do seu corpo, onde se alimenta e empupa. Quando perturbada, produz um ruído, possivelmente, na tentativa de afastar o inimigo. Foi registrada principalmente nos meses de Setembro e Outubro de 2004. Não foram encontrados registros de plantas hospedeiras disponíveis para essa espécie.

Noctuidae. Paectes albescens Hampson, 1912 (Fig. 10): Possui coloração esverdeada com pigmentos verdes claros, e uma linha também de coloração esverdeada, na parte inferior do corpo. Alimenta-se livremente nas folhas, ficando na superfície abaxial até empupar no solo. Encontrada, apenas, em P. icicariba, nos meses de Novembro de 2004 até Março de 2005. Não foram encontrados registros de plantas hospedeiras para essa espécie.

Paectes songoensis Zerny, 1916 (Fig. 11): Possui coloração verde escura com linhas brancas longitudinais no seu corpo. Apresenta vida livre e alimenta-se na superfície abaxial das folhas, empupando no solo. Foi encontrada em $P$. icicariba nos meses de Agosto e Setembro de 2004 e em P. heptaphyllum nos meses de Novembro de 2004 à Janeiro de 2005 e em Abril de 2005. Não foram encontrados registros de plantas hospedeiras para essa espécie.

Paectes semicircularis Hampson, 1912 (Fig. 12): Com coloração verde, possui cinco linhas transversais de coloração verde claro ao longo do corpo. Alimenta-se na parte inferior das folhas, apresentando comportamento de vida livre e empupa no solo. Foi encontrada em $P$. heptaphyllum, nos meses de Novembro de 2004, Março e Abril de 2005. Não foram encontrados registros de plantas hospedeiras para essa espécie.

Notodontidae. Rifargia causia (Schaus, 1892) (Fig. 13): Possui o corpo com coloração esverdeada com detalhes em marrom. Alimenta-se na parte abaxial das folhas e empupa no solo. Foi encontrada em P. icicariba, apenas, no mês de Novembro de 2004. Não foram encontrados registros de plantas hospedeiras para essa espécie.

Rifargia distinguenda (Walker, 1856) (Fig. 14): Coloração 
predominantemente amarronzada com manchas verde claras. Alimenta-se na parte abaxial das folhas e empupa no solo. Foi encontrada em P. icicariba nos meses de Junho e Outubro de 2004. Existem registros dessa espécie alimentando-se de Anacardium excelsum (Bael et al. 2004), Tapirira mexicana (Anacardiaceae) e Bursera simaruba (Burseraceae) (Janzen \& Hallwachs 2006).

Oecophoridae. Inga inflammata (Meyerick, 1916) (Fig. 15): Coloração marrom claro, fazendo abrigo com seda, folhas e frutos, onde vive, se alimenta e empupa. Polífaga, no presente estudo foi encontrada em Erythroxylum subsessile, E. ovalifolium, Manilkara subsericea e Protium icicariba, sendo registrada nos meses de Julho e Agosto de 2004 e Abril e Maio de 2005. Flinte et al. (2006) também registraram essa espécie alimentando-se de Byrsonima sericea (Malpighiaceae).

Pyralidae. Zamagiria laidion (Zeller, 1881): Cor verde clara, as larvas dessa espécie formam um abrigo, envolvendo com seda suas fezes e três ou quatro folhas, dentro do qual agregamse cinco ou mais larvas. Seu pico de abundância foi registrado em dezembro de 2003. Essa espécie foi registrada também em Manilkara emarginata, Manilkara jaimiqui e Mimusops elengi (Sapotaceae) (Robinson 1999), além de atacar plantações de sapoti (Manilkara zapota: Sapotaceae) (Teran 1980; Iruegas et al. 2002; Carneiro et al. 2003), o que sugere seu hábito oligófago.

Sphingidae. Erinnyis ello ello Linnaeus, 1758: Coloração verde claro, com um ocelo azul escuro na parte dorsal do corpo. Quando perturbada mostra um comportamento deimático, expondo o seu ocelo. Apenas um indivíduo dessa espécie foi coletado, no mês de Março de 2004. Alimenta-se, principalmente, de plantas pertencentes à família Euphorbiaceae, como Euphorbia gymnoclada, E. prostrata, E. pulcherrima, E. cyathophora, E. heterophylla, E. mesembrianthemifolia, Hevea brasiliensis, Manihot esculenta, M. glaziovii, Ricinus communis, Sapium aucuparium, Jatropha palmata, Cnidoscolus angustidens (Robinson 1999). Entretanto, existem registros também em Chrysophyllum oliviforme, Sideroxylon celastrinum, S. salicifolium, S. tepicense (Sapotaceae), Allamanda cathartica (Apocynaceae), Araujia sericofera (Asclepiadaceae), Arachis hypogaea (Leguminosae), Lycopersicon esculentum (Solanaceae), Carica papaya (Caricaceae) e Psidium guajava (Myrtaceae) (Robinson 1999), o que a caracteriza como polífaga.

Protambulyx eurycles (Herrich-Schäffer, 1854) (Fig. 16): Possui coloração verde claro com listras brancas ao longo do corpo e um prolongamento no final do mesmo. Alimenta-se na superfície das folhas, camuflando-se ao longo da nervura, e empupa no solo. Foi encontrada em P. icicariba, apenas no mês de Junho de 2004. Existem registros dessa espécie alimentando-se de Spondias dulcis, S. mombin, Astronium graveolens (Anacardiaceae), e Simarouba glauca e S. amara (Simaroubaceae) (Oehlke 2006).
Thyrididae. Banisia myrsusalis (Walker, 1859): Coloração clara, com o tegumento translúcido. Vive e alimenta-se dentro de um abrigo formado pela união com sedas das extremidades de uma única folha nova. Ao terminar o seu desenvolvimento, sai da sua planta hospedeira e empupa no solo. Ocorreu nos meses de Outubro de 2003 e Abril e Maio de 2004. Possui hábito alimentar, possivelmente, oligófago, uma vez que existem registros dessa espécie atacando plantações de sapotizeiros (Manilkara zapota) (Silva et al. 2003), Chrysophyllum oliviforme (Bendicho-Lopez 1998), C. cainit, Madhuca indica, M. latifolia, M. longifolia, Mimusops elengi e Pouteria caimito (Robinson 1999), todas pertencentes à família Sapotaceae.

Das 32 espécies de Lepidoptera exofíticas encontradas nas cinco espécies de plantas hospedeiras, $56 \%(\mathrm{n}=18)$ vivem no interior de algum tipo de abrigo. Para a construção desses abrigos são utilizadas, principalmente, folhas unidas com seda. Esse tipo de proteção pode ser importante na defesa contra inimigos naturais (Jones et al. 2002), mas também pode ser uma adaptação fundamental para evitar a sua dessecação (Diniz et al. 2000). Flinte et al. (2006), estudando a entomofauna associada à Byrsonima sericea (Malpighiaceae) na mesma área de restinga encontraram que 70\% das espécies de Lepidoptera constroem abrigos, enquanto que Diniz et al. (2000), encontraram que $61 \%$ das larvas de Lepidoptera amostradas em um cerrado vivem em abrigos. Essa alta proporção de espécies utilizando-se de abrigos pode estar relacionada, no caso da restinga aberta, principalmente, à necessidade de proteção contra dessecação, provocada pela insolação e ventos fortes, freqüientes nesse ambiente.

Somente Zamagiria laidion, apresentou hábito gregário e apenas três espécies, Megalopyge lanata, Phobetron hipparchia e Trichromia onytes, eram densamente pilosas. Esse baixo número de espécies com esses dois tipos de defesas pode ser explicado pelo fato de grande parte das espécies amostradas alimentarem-se e viverem "protegidas" no interior de abrigos.

Um dos principais temas abordados na literatura na última década é a especificidade dos insetos fitófagos (Price et al. 1995; Diniz \& Morais 1997; Novotny \& Basset 2000; Flinte et al. 2006). Essa especificidade é tratada em uma escala ampla, ao nível da espécie, ou local, quando somente uma população da espécie é considerada. Assim, analisando-se a especificidade geral das espécies identificadas, levando-se em consideração os dados do presente estudo e aqueles encontrados na literatura (inclusive e principalmente oriundos de outras localidades), foram encontradas seis espécies (27\%) polífagas, três (14\%) oligófagas e 13 (59\%) monófagas. Paectes songoensis pode ser considerada uma espécie oligófaga local, pois, foi encontrada nas duas espécies de Protium estudadas no PNRJ. Banisia myrsusalis e Zamagiria laidion, ambas com registros, na literatura, em diferentes espécies de Sapotaceae, são consideradas oligófagas gerais, e muito embora tenham sido encontradas, apenas, em M. subsericea podem também ocorrer em outras espécies de Sapotaceae, não amostradas, na área de estudo. Já Erinnyis ello ello, Protambulyx eurycles 
e Rifargia distinguenda embora encontradas alimentando-se de apenas uma espécie de planta hospedeira devem ser polífagas, mesmo ao nível local, uma vez que apresentam registros, na literatura, em diversas plantas pertencentes à famílias distintas. As espécies Inga inflammata, Megalopyge lanata e Phobetron hipparchia são polífagas locais, pois, no presente estudo, foram encontradas alimentando-se de plantas pertencentes a diferentes famílias.

Das 13 espécies classificadas como monófagas, apenas quatro foram abundantes em suas plantas hospedeiras, apresentando mais de 50 indivíduos a cada 100 plantas vistoriadas, foram elas: Chlamydastis fragmentella, Stenoma sp.1, Stenoma sp.2 e Stenoma sp.4 (Tabela I). Essas espécies de Elachistidae devem ser de fato monófagas locais, uma vez que são abundantes e não foram encontradas, até o momento, em nenhuma outra espécie de planta na restinga. As outras nove espécies foram bastante raras nas plantas hospedeiras vistoriadas. Entretanto, segundo Novotny \& Basset (2000), essas espécies poderiam estar se alimentando ocasionalmente na planta estudada, mas prefeririam outras plantas hospedeiras, onde seriam mais abundantes. Assim, a especificidade encontrada no presente estudo, para essas nove espécies, poderia ser apenas aparente, devido a um artefato de amostragem.

Para as espécies de Lepidoptera mais abundantes, foi encontrado um padrão de distribuição temporal onde os picos de abundância ocorreram antes dos picos de folhas novas de suas plantas hospedeiras (Figs. 17 a 20). A produção de folhas novas pelas plantas hospedeiras estudadas ocorreu, principalmente, entre os meses de Setembro e Dezembro, que corresponde à primavera; enquanto que os picos de abundância das Lepidoptera ocorreram entre Março e Abril ou entre Julho e Setembro. Trabalhos anteriores, realizados na restinga (Flinte et al. 2006) e no cerrado (Morais et al. 1999), mostraram padrão semelhante de distribuição temporal de Lepidoptera. Morais et al. (1999) sugerem que esse pico antes da produção de folhas novas pode ser uma estratégia de fuga de inimigos naturais. Esses autores possuem evidências de que na época úmida, quando ocorre maior produção de folhas novas, acontecem também maiores taxas de parasitismo sobre as larvas. Por esse motivo, essas espécies antecipariam seu período reprodutivo, apresentando picos de abundância antes do pico de folhas novas.

Embora não possamos descartar a possibilidade dos inimigos naturais moldarem o padrão temporal dos insetos fitófagos, consideramos que fatores abióticos exerçam papel importante na restinga. Nesse ecossistema, de vegetação aberta e solo arenoso, durante a primavera e verão, época mais chuvosa, ocorre também forte insolação, no intervalo dos períodos de chuva, o que aumenta grandemente o risco de dessecação para as plantas e insetos.

Agradecimentos. Ao Dr. Victor Becker pela identificação das espécies de Lepidoptera e a Dra. Dorothy S.D. de Araújo pela confirmação da identificação das espécies de plantas. Viviane Grenha fez algumas observações sobre Phobetron hipparchia. Aos dois revisores desse artigo que contribuíram para a melhoria geral do texto. Este trabalho faz parte do Programa de Pesquisas Ecológicas de Longa Duração (PELD / Site 5), financiado pelo CNPq. Ao CNPq também pelo apoio das bolsas de iniciação científica (Milena de S. Nascimento) e de produtividade em pesquisa (Ricardo F. Monteiro) e ao IBAMA pela licença para pesquisa no PNRJ.

\section{REFERÊNCIAS}

Andrade, I.; H. C. Morais; I. R. Diniz \& C. Van Den Berg. 1999. Richness and abundance of caterpillars on Byrsonima (Malpighiaceae) species in an area of cerrado vegetation in Central Brazil. Revista de Biología Tropical 47: 691-695.

Araújo, D. S. D.; F. R. Scarano; C. F. C. Sá; B. C. Kurtz; H. L. T. Zaluar; R. C. M. Montezuma \& R. C. Oliveira. 1998. Comunidades vegetais do Parque Nacional da Restinga de Jurubatiba, p.39-62. In: F.A. Esteves (ed.). Ecologia de Lagoas Costeiras do Parque Nacional da Restinga de Jurubatiba e do Município de Macaé (RJ). Rio de Janeiro, UFRJ/NUPEM, 442 p.

Araújo, D. S. D.; M. C. A. Pereira \& M. C. P. Pimentel. 2004. Flora e Estrutura de Comunidades na Restinga de Jurubatiba - Síntese dos Conhecimentos com Enfoque Especial para a Formação Aberta de Clusia, p.59-76. In: C. F. D. Rocha; F. A. Esteves; F. R. Scarano. Pesquisas de Longa Duração na Restinga de Jurubatiba Ecologia, História Natural e Conservação. São Carlos, RiMa Editora, 374 p.

Bael, S. A. V.; A. Aiello; A. Valderrama; E. Medianero; M. Samaniego \& S. J. Wright. 2004. General herbivore outbreak following an El Niño-related drought in a lowland Panamanian forest. Journal of Tropical Ecology 20: 625-633.

Bendicho-Lopez, A. C. 1998. New distribution and food plant records for twenty cuban moths. Journal of the Lepidopterists' Society 52: 214-216.

Carneiro, J. S.; P. H. S. Silva \& H. U. Sousa. 2003. Ocorrência de Zamagiria laidion (Lepidoptera: Pyralidae) atacando sapotizeiros na Região dos Tabuleiros Costeiros do Piauí. Embrapa: Comunicado Técnico 156: 1-3.

Costa Lima, A. 1945. Insetos do Brasil - Lepidópteros - Série Didática $\mathbf{n}^{\circ} \mathbf{7}-\mathbf{5}^{\circ}$ tomo. Rio de Janeiro, Escola Nacional de Agronomia, 379 p.

Costa Lima, A. 1950. Insetos do Brasil - Lepidópteros - Série Didática $\mathbf{n}^{\circ} 8-\mathbf{6}^{\mathbf{0}}$ tomo. Rio de Janeiro, Escola Nacional de Agronomia, $420 \mathrm{p}$.

D’Araújo e Silva, A. G.; C. R. Gonçalves; D. M. Galvão; A. J. L. Gonçalves; J. Gomes; M. N. Silva \& L. Simoni. 1968. Quarto Catálogo dos Insetos que Vivem nas Plantas do Brasil, seus Parasitos e Predadores - ParteII - 1'Tomo. Rio de Janeiro, Fundação IBGE, $622 \mathrm{p}$.

Diniz, I. R. \& H. C. Morais. 1995. Larvas de Lepidoptera e suas plantas hospedeiras em um cerrado de Brasília, DF, Brasil. Revista Brasileira de Entomologia 39: 755-770.

Diniz, I. R. \& H. C. Morais. 1997. Lepidopteran caterpillar fauna of Cerrado host plants. Biodiversity and Conservation 6: $817-$ 836.

Diniz, I. R.; H. C. Morais; A. M. F. Botelho; F. Venturoli \& B. C. Cabral. 1999. Lepidopteran caterpillar fauna on lactiferous host plants in the Central Brazilian Cerrado. Revista Brasileira de Biologia 59: 627-635.

Diniz, I. R.; H. C. Morais \& J. D. Hay. 2000. Natural history of herbivores feeding on Byrsonima species. Brazilian Journal of Ecology 1 e 2: 49-54.

Ehrlich, P. R. \& P. H. Raven. 1964. Butterflies and plants: a study in coevolution. Evolution 18: 586-608.

Flinte, V. \& M. V. Macedo. 2004. Biology and Seasonality of Fulcidax monstrosa (F.) (Chrysomelidae: Chlamisinae). The Coleopterists Bulletin 58: 457-465.

Flinte, V.; C. O. Araujo; M. V. Macedo \& R. F. Monteiro. 2006. Insetos fitófagos associados ao murici da praia, Byrsonima sericea (Malpighiaceae), na Restinga de Jurubatiba (RJ). Revista 
Brasileira de Entomologia 50: 512-523.

Iruegas, R.; B. Gomez; L. Cruz-Lopes; E. A. Malo \& J. C. Rojas. 2002. A new record of a moth attacking sapodilla, with descriptions of female genitalia and the last instar larva. Florida Entomologist 85: 394-397.

Janzen, D. H. \& Hallwachs, W. 2006. Philosophy, navigation and use of a dynamic database ("ACG Caterpillars SRNP") for an inventory of the macrocaterpillar fauna, and its food plants and parasitoids, of Area de Conservacion Guanacaste (ACG), northwestern Costa Rica. (http://janzen.sas.upenn.edu), acesso em: 18/03/2006.

Jones, M. T.; I. Castellanos \& M. R. Weiss. 2002. Do leaf shelters always protect caterpillars from invertebrate predators? Ecological Entomology 27: 753-757.

Lawton, J. H. 1982. Vacant niches and unsaturated comunities: a comparison of bracken herbivores at sites on two continents. Journal of Animal Ecology 51: 573-595.

Leather, S. R. 1986. Insect species richness of the British Rosaceae: the importance of host range plant, architecture, age of establishment, taxonomic isolation and species-area relationships. Journal of Animal Ecology 55: 841-860.

Martius, K. P. von; A. G. Eichler \& I. Urban. 1874. Flora brasiliensis - Volume 12 - Parte 2. p. 262-264.

Monteiro, R. F. \& M. V. Macedo. 2000. Flutuação Populacional de Insetos Fitófagos em restinga, p.77-88. In: F. A. Esteves \& L. D. Lacerda (eds.) Ecologia de Restingas e Lagoas Costeiras. Rio de Janeiro, UFRJ/NUPEM, 446 p.

Monteiro, R. F. \& V. O. Becker. 2002. A new Sigelgaita Heinrich (Lepidoptera, Pyralidae, Phycitinae) feeding on cacti in Brazil. Revista Brasileira de Zoologia 19: 299-304.

Monteiro, R. F.; A. P. Esperanço; V. O. Becker; L. S. Otero; E. V. Herkenhoff \& A. Soares. 2004. Mariposas e Borboletas na Restinga de Jurubatiba, p.143-164. In: C.F.D. Rocha, F.A. Esteves, F.R. Scarano (eds.). Pesquisas de Longa Duração na Restinga de Jurubatiba - Ecologia, História Natural e Conservação. São Carlos, RiMa Editora, 374 p.
Morais, H. C.; I. R. Diniz \& D. M. S. Silva. 1999. Caterpillar seasonality in a central Brazilian cerrado. Revista de Biología Tropical 47: 1025-1033.

Morais, H. C.; I. M. Mahajan \& I. R. Diniz. 2005. História natural da mariposa Chlamydastis smodicopa (Meyrick) (Lepidoptera, Elachistidae, Stenomatinae). Revista Brasileira de Zoologia 22: 633-638.

Novotny, V. \& Y. Basset. 2000. Rare species in communities of tropical insect herbivores: pondering the mystery of singletons. Oikos 89: 564-572.

Oehlke, B. 2006. Sphingidae of the Americas (http:// www.silkmoths.bizland.com/danjansphinx.htm). Acesso em: 18/03/ 2006.

Pinheiro, F.; I. R. Diniz; D. Coelho \& M. P. S. Bandeira. 2002. Seasonal pattern of insect abundance in the Brazilian cerrado. Austral Ecology 27: 132-136.

Price, P. W.; I. R. Diniz; H. C. Morais \& E. S. A. Marques. 1995. The abundance of insect herbivore species in the tropics: high local richness of rare species. Biotropica 27: 468-478.

Robinson, G. S. 1999. HOSTS - a database of the hostplants of the world's Lepidoptera. Nota Lepidopterologica 22: 35-47.

Silva, P. H. S.; J. S. Carneiro \& H. U. Sousa. 2003. Ocorrência de Banisia myrsusalis (Walker, 1895) atacando sapotizeiros na Região dos Tabuleiros Costeiros do Piauí. Embrapa: Comunicado Técnico 157: $1-3$

Southwood, T. R. E. 1973. The insect/plant relationship - an evolutionary perspective, p. 3-29. In: H. F. Van Emden (eds.). Insect/Plant Relationships. Oxford, Blackwell, 215 p.

Southwood, T. R. E. 1986. Plant surfaces and insects - an overview, p.1-22. In: B. Juniper \& R. Southwood (eds.). Insects and the Plant Surface. Baltimore, Hodder Arnold, 368 p.

Teran, J. B. 1980. Lista preliminar de Hymenoptera parasitos de otros insectos en Venezuela. Revista de la Facultad de Agronomía (Maracay) 11: 283-389.

Young, A. M. 1986. Notes on a Costa Rican "Monkey Slug" (Limacodidae). Journal of the Lepidopterists' Society 40: 69-71. 